\title{
Phase-field simulations of lithium dendrite growth with open-source software
}

\author{
Zijian Hong and Venkatasubramanian Viswanathan* \\ Department of Mechanical Engineering, Carnegie Mellon University, Pittsburgh, \\ Pennsylvania 15213, USA \\ E-mail: venkvis@cmu.edu
}

\begin{abstract}
Dendrite growth is a long-standing challenge that has limited the applications of rechargeable lithium metal electrodes. Here, we have developed a grand potential based nonlinear phase-field model to study the electrodeposition of lithium as relevant for a lithium metal anode, using open-source software package MOOSE. The dynamic morphological evolution under large/small overpotential is studied in 2-dimensions, revealing important dendrite growth/stable deposition patterns. The corresponding temporal-spatial distributions of ion concentration, overpotential and driving force are studied, which demonstrate an intimate, dynamic competition between ion transport and electrochemical reactions, resulting in vastly different growth patterns. Given the importance of morphological evolution for lithium metal electrodes, wide-spread applications of phase-field models have been limited in part due to in-house or proprietary software. In order to spur growth of this field, we utilize an open-source software package and make all files available to enable future studies to study the many unsolved aspects related to morphology evolution for lithium metal electrodes.
\end{abstract}


There is a need for increasing the energy density of batteries for enabling a complete electrification of transportation. ${ }^{1 / 3}$ Lithium metal anodes represent one of the most promising near-term solutions enabling increased energy density. ${ }^{\underline{4}}$ The main challenge with lithium metal anodes stems from dendrite growth during electrodeposition, which leads to deposits that could penetrate through the separator, resulting in safety issues and coloumbic inefficiency. ${ }^{[5]}$ A comprehensive understanding of the morphology evolution during the electrodeposition process is of paramount importance for the design of next-generation batteries with Lithium metal anode.

Most of the previous theoretical investigations of dendrite formation during electrodeposition is based on linear stability analysis, originally pioneered by Mullins and Sekerka,, 910 where the thermodynamic stability of a small perturbation is studied in terms of a change in the chemical potential. $11+15$ This simple approach has revealed the impact of some key physical parameters (elastic properties, molar volume, etc. ${ }^{14}[16)$. However, the linear stability analysis approach is limited to the understanding of dendrite initiation and does not take into account effects beyond nucleation, dimensionality of growth and dynamics of the growth process. In particular, the key features related to morphology evolution in three-dimensions are missed within this approach.

Phase-field modeling is a mesoscale simulation tool that enable the quantitative understanding of phase transitions, phase transformations and microstructure evolutions. ${ }^{17118}$ To date, several phase-field models have been built to model the dynamic evolution of dendrite growth during the electrodeposition process, incorporating the nonlinear electrochemical reaction dynamics. $\frac{19}{24}$ However, these studies typically utilize custom-built software or proprietary code which could hinder the wide-spread applications of phase-field models, while also makes it extremely difficult for follow-on works and reproducible science. ${ }^{25}$

Herein, we developed a hybrid grand potential based nonlinear phase field model, 19120126 and numerically solving the multiphysics coupled equations using the fully open source MOOSE (Multiphysics Object-Oriented Simulation Environment) framework. ${ }^{[27}$ This enables 
a quantitative and comprehensive understanding of the morphological evolution during the electrodeposition process. The well tested and documented MOOSE package allows for fast, parallel and customized numerical modeling, which is easy to follow and simple to implement and validate. Most importantly, it has an already built-in, well-benchmarked phase-field module that could be utilized for further implementations. ${ }^{28299}$ In this work, the dynamic morphological evolution for lithium deposition is studied, where both stable deposition and dendrite growth are captured at low and high applied overpotentials, respectively. The corresponding spatial-temporal distributions of concentration, over-potential and driving force are studied in details, which reveals an intimate, dynamic competition between lithium-ion transport (from both ion diffusion and electrical migration) and electrochemical reaction, resulting in complete different growth patterns. A concept of "compositionally graded solid electrolyte" is proposed, which could provide an approach to suppress dendrite initiation based on the insights from this study. We believe that this study will spur on the application of phase-field models that can handle more complicated problems incorporates other effects (elastic effect, solid electrolyte interfaces, etc.).

Phase field model. The phase field variable $\xi$ is used as the non-conserved order parameter which is defined such that $\xi=1$ and $\xi=0$ for the pure electrode and electrolyte phases, respectively. Following the derivations by Chen et al. $\frac{19}{19}$ the temporal evolution of the order parameter $\xi$ can be expressed as:

$$
\frac{\partial \xi}{\partial t}=-L_{\sigma}\left(g^{\prime}(\xi)-k \nabla^{2} \xi\right)-L_{\eta} h^{\prime}(\xi)\left\{\exp \left[\frac{(1-\alpha) n F \eta_{\alpha}}{R T}\right]-\frac{c_{L i^{+}}}{c_{0}} \exp \left[\frac{-\alpha n F \eta_{\alpha}}{R T}\right]\right\}
$$

where $L_{\sigma}$ and $L_{\eta}$ are the interfacial mobility and electrochemical reaction kinetic coefficient, respectively. $g(\xi)$ is the double well function, expressed by $W \xi^{2}(1-\xi)^{2}$. $W$ is related to the switching barrier ${ }^{19} k, \alpha, n, F, R, t$ and $T$ are the gradient coefficient, charge transfer coefficient, number of electrons transfered, Faraday constant, gas constant, evolution time and temperature, respectively. The switching barrier and gradient coefficient are further 
related to the surface tension $\gamma$ and interfacial thickness $\delta$, i.e. $W=\frac{3 \gamma}{\delta}$ and $k=6 \gamma \delta \underline{1823}$ Whereas $h(\xi)=\xi^{3}\left(6 \xi^{2}-15 \xi+10\right)$ is the interpolation function. $\frac{19}{\eta_{\alpha}}$ is the activation overpotential $\eta_{\alpha}=\phi-E^{\theta}, E^{\theta}$ is the standard equilibrium half cell potential, $\phi$ is the applied overpotential. $c_{L^{+}}$and $c_{0}$ are the local and initial lithium-ion molar ratio. In a two phase model, the local lithium ion molar-fraction can be written as: $\underline{20}$

$$
c_{L i^{+}}=c^{l}(1-h(\xi))=\frac{\exp \left[\frac{\left(\mu-\epsilon^{l}\right)}{R T}\right]}{1+\exp \left[\frac{\left(\mu-\epsilon^{l}\right)}{R T}\right]}(1-h(\xi)) .
$$

where $c^{l}$ is the molar fraction of lithium in the electrolyte phase, $\mu$ is the chemical potential of lithium. $\epsilon^{l}=\mu^{0 l}-\mu^{0 N}$ is the differences in the chemical potential of lithium and neutral components on the electrolyte phase at initial equilibrium state.

The chemical potential can be obtained by solving the modified diffusion equation 20 (detailed derivation is given in Supplementary Information):

$$
\frac{\partial \mu}{\partial t}=\frac{1}{\chi}\left[\nabla \cdot \frac{D c_{L i^{+}}}{R T}(\nabla \mu+n F \nabla \phi)-\frac{\partial h(\xi)}{\partial t}\left(c^{s} \frac{C_{m}^{s}}{C_{m}^{l}}-c^{l}\right)\right]
$$

where $C_{m}^{s}$ and $C_{m}^{l}$ are the site density of the electrode and electrolyte phases (inverse of molar volume), respectively. $c^{s}$ is the molar fraction of lithium in the electrode phase, while $\chi=\frac{\partial c^{l}}{\partial \mu}[1-h(\xi)]+\frac{\partial c^{s}}{\partial \mu} h(\xi) \frac{C_{m}^{s}}{C_{m}^{l}}$.

The spatial distribution of the electrical overpotential $\phi$ can be obtained by solving the conduction equation:

$$
\nabla \sigma \nabla \phi=n F C_{m}^{s} \frac{\partial \xi}{\partial t}
$$

The effective conductivity $\sigma$ is related to the conductivity of the electrode phase $\sigma^{s}$ and electrolyte phase $\sigma^{l}$, i.e., $\sigma=\sigma^{s} h(\xi)+\sigma^{l}[1-h(\xi)]$

Numerical simulations. Equations 1-4 are solved using the open source MOOSE framework. A two-dimensional mesh of $200 \times 200$ is used, with each grid representing $1 \mu \mathrm{m}$. The simulation temperature is $300 \mathrm{~K}$. The Preconditioned Jacobian Free Newton Krylov 
(PJFNK) method is set as the solve type, with a bdf2 scheme and Single Matrix Preconditioning (SMP). A timestep interval $(\mathrm{dt})$ of $0.02 \mathrm{~s}$ is set, with a maximum simulation time of $400 \mathrm{~s}$. The key simulation parameters before and after normalization are listed in Supplementary table 1 . The normalization constants for length, time and energy scales are set as $1 \mu \mathrm{m}, 1 \mathrm{~s}$ and $2.5 \times 10^{6} \mathrm{~J} / \mathrm{m}^{3}$. The standard equilibrium half cell potential is set to be 0 for the sake of convenience. A Langevin noise level of 0.04 is added (corresponding to $0.056 \%$ change in magnitude) to the order parameter $\xi$ to stimulate the thermal fluctuation in the system. The Neumann boundary condition is applied at the $X$-dimension, such that the chemical potential is fixed to be zero at both sides (indicating a constant Lithium molar ratio at the two ends), while the order parameter is set as 1 (electrode phase) and 0 (electrolyte phase) at the left and right sides, respectively. The electric overpotential is fixed to the applied overpotential at the electrode side and zero at the electrolyte side to give a constant activation overpotential across the electrode/electrolyte interface. The no flux boundary condition is applied along the $Y$-dimension for all primary variables $(\xi, \mu$ and $\phi)$. A set of applied electric overpotential ranging from $-0.3 \mathrm{~V}$ to $-0.5 \mathrm{~V}$ is studied.

Results. The lithium growth kinetics under an applied overpotential of $-0.45 \mathrm{~V}$ is shown in Figure 1, assuming a $\mathrm{Li}^{+}$concentration of $1 \mathrm{M}$ in a standard carbonate-based electrolyte. The initial structure is shown in Figure 1(a), an electrode with a thickness of $20 \mu \mathrm{m}$ is separated with the electrolyte by a smooth interface. No initial nuclei are added, the dendrite initiation and growth is a purely intrinsic process in this study. This differs from several previous studies where the simulation results might be sensitive to the preset artificial nuclei size, shape and orientation. $\frac{1922129}{29}$ After an electrodeposition process of $57 \mathrm{~s}$ (Figure1 $1 \mathrm{p}$ ), the interface moves towards the electrolyte by $\sim 20 \mu \mathrm{m}$. The interfacial instability is discovered, with a modulation of surface roughness for a vertical period of $\sim 10 \mu \mathrm{m}$. Further growth of these nuclei leads to the formation of long needle-like dendrites (Figure 1c) with a length of $\sim 50 \mu \mathrm{m}$, while the diameter of the dendrites only increases by $\sim 10 \mu \mathrm{m}$, indicating a quasi-directional growth. A short video of the dendrite growth process under this condition 
is given in Supplementary video 1. It is worthwhile noting that although $\sim 20$ nuclei is formed initially, only a few of them can grow into dendrites. The corresponding evolution
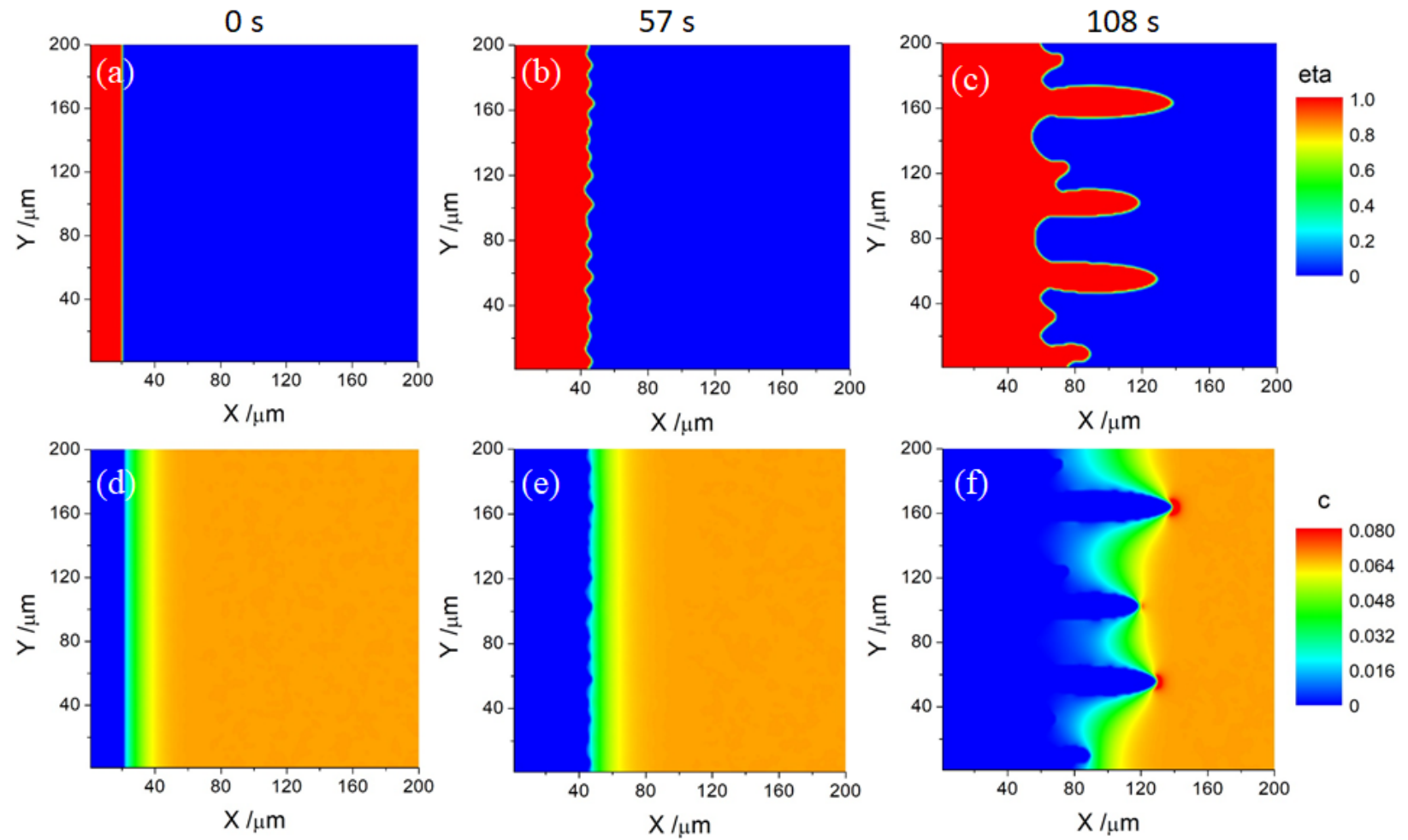

Figure 1: Dendrite growth and concentration evolution with applied overpotential of $-0.45 \mathrm{~V}$. (a)-(c) Morphology evolution after Lithium electrodeposition of 0 s, 57 s and 108 s. (d)-(f) Corresponding evolution of Lithium-ion molar ratio.

of $\mathrm{Li}^{+}$ion molar fraction is shown in Figures $1(\mathrm{~d})-(\mathrm{f})$. It is revealed that a broad interfacial region (shown in green) is formed to separate the electrode (with zero $\mathrm{Li}^{+}$molar ratio) and the electrolyte (with a homogeneous $\mathrm{Li}^{+}$molar ratio of $\sim 0.067$ ). With the formation of small nuclei at the interface (Figure 11), a small fluctuation of ion concentration is also formed accordingly where the tip region has a slightly higher concentration than the valley region. The further growth of dendrites leads to a higher $\mathrm{Li}^{+}$concentration surrounding the dendrite tips, with a lower ion concentration formed in the valley regions. The enrichment of $\mathrm{Li}^{+}$molar ratio in the vicinity of the dendrite tips is due to the shortened diffusion path compared to the valley regions.

In order to take a deeper understanding of the dendrite growth mechanism, the spatial- 
temporal distribution of electric potential and driving force is plotted in Figure2. As shown in Figure 2(a), the electric potential is nearly constant in the electrode region, followed by a gradual, linear increase from the electrode/electrolyte interface to the electrolyte region (see Figure S2d). After $57 \mathrm{~s}$ (Figure 2b), as the interface is moving towards the electrolyte region, the gradient of electric potential (electric field) increases. The electric field is still more or less homogeneous inside the electrolyte region. With the growth of dendrite (Figure 22), a lower electric potential is found near the tip as compared to the surrounding valley regions, thus, we expect not only a large electric field along the $X$-dimension, but also an electric field towards the dendrite tip along the $Y$-dimension as well (shown in Figure S3). This could further facilitate $\mathrm{Li}^{+}$transport from both the source and the surrounding valley regions to the dendrite tips, leading to an "entrainment" phenomenon. The driving force (defined as $\left.L_{\eta} h^{\prime}(\xi)\left\{\exp \left[\frac{(1-\alpha) n F \eta_{\alpha}}{R T}\right]-\frac{c_{L i+}}{c_{0}} \exp \left[\frac{-\alpha n F \eta_{\alpha}}{R T}\right]\right\}\right)$ for the electrochemical reaction is directly related to the interface growth velocity $\frac{\partial \xi}{\partial t}$. As depicted in Figure 2(d), there's a large driving force at the electrode/electrolyte interface, which can be understood since the electrochemical reaction only occurs at the interface where $h^{\prime}(\xi)$ is non-zero. After $57 \mathrm{~s}$ (Figure 2e), with the continuous electrochemical deposition, the driving force at the interface increases, while forming a zig-zag like pattern, mimicking the modulation of the solid/liquid interface. With the growth of the dendrites after $108 \mathrm{~s}$ (Figure 2f), it is clearly shown that the driving force at the dendrite tips are orders of magnitude larger than the interface of the valley regions. Whereas the longer the dendrite, the larger the driving force, which further facilitates growth of the longer dendrites. Figure $2(\mathrm{~g})$ and $(\mathrm{h})$ show the line plots for the time evolution of Li-ion molar fraction along $X$-direction cutting through the valley and dendrite regions, respectively. It can be seen that up until $57 \mathrm{~s}$ where the dendrites are yet to grow, the concentration profile in the two regions are very similar, the interface region has a lower $\mathrm{Li}^{+}$ ion molar fraction as compared to the bulk. As the dendrites grow longer (e.g., $96 \mathrm{~s}$ ), we see an enrichment/depletion of ion concentration at the dendrite/valley regions, resulting in a widely broadened interface for the valley region which can hardly move thereafter. The 
line plots of the driving force for the two regions are shown in figure 2(i) and (j). Spikes are found at the interfaces in accordance to the concentration profiles. After $57 \mathrm{~s}$, as the interface is moving towards the electrolyte, the electric field increases, which increases the driving force. Eventually, the growth of dendrites will lead to a huge increase in the driving force which can be partly attributed to the increase of ion concentration surrounding the dendrite tip; whereas the valley region gets an order of magnitude suppression in the driving force, resulting from the depletion of $\mathrm{Li}^{+}$ion.

The morphology evolution at low applied overpotential, e.g., $-0.32 \mathrm{~V}$ is further studied to make a detailed comparison (Figure 3). A stable electrochemical deposition is observed with no obvious surface modulation (Figure 3a). Interestingly, near the electrode/electrolyte interface, an enrichment in the $\mathrm{Li}^{+}$ion is discovered (Figure 3b). This can be understood since the deposition velocity is greatly reduced (exponentially) at lower overpotential, while the ion transport only depends on the gradient of the potential distribution which roughly decreases linearly with decreasing applied overpotential. The temporal evolution of the maximum $\mathrm{Li}^{+}$concentration near the interface is plotted in Figure 3(c). An oscillation of the maximum $\mathrm{Li}^{+}$ion molar ratio is captured, indicating a dynamic competition between the ion transport and electrochemical reaction. The line plot of the average $\mathrm{Li}^{+}$concentration profile after passing the same total current is plotted for different overpotentials (Figure 3d), a gradual shift from surface concentration enrichment to surface concentration depletion is observed from lower to higher overpotentials $(0.32 \mathrm{~V}-0.5 \mathrm{~V})$. And the threshold overpotential for dendrite growth occurs exactly at the transition point from interface concentration enrichment to depletion $(\sim 0.37 \mathrm{~V})$.

Discussions. The schematics of the two scenarios are given below for a discussion. At lower applied overpotential, initially, the reaction rate is much smaller than the ion transport rate (including diffusion and migration), resulting in an accumulation of ion concentration at the interface, which would in turn boost the reaction rate (since it is directly proportional to the ion concentration) while decreasing the transport rate (due to a large negative con- 

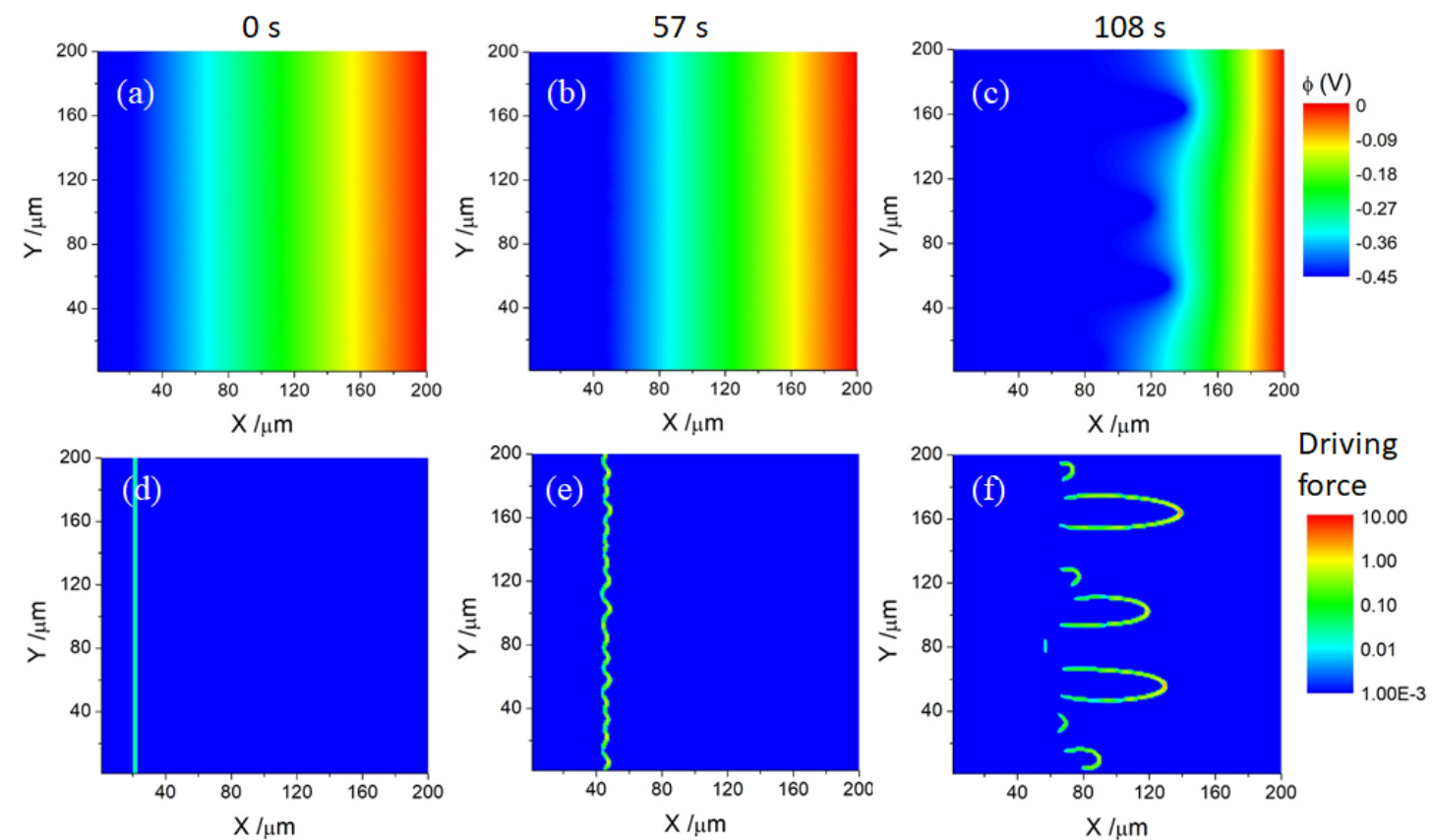

Valley region
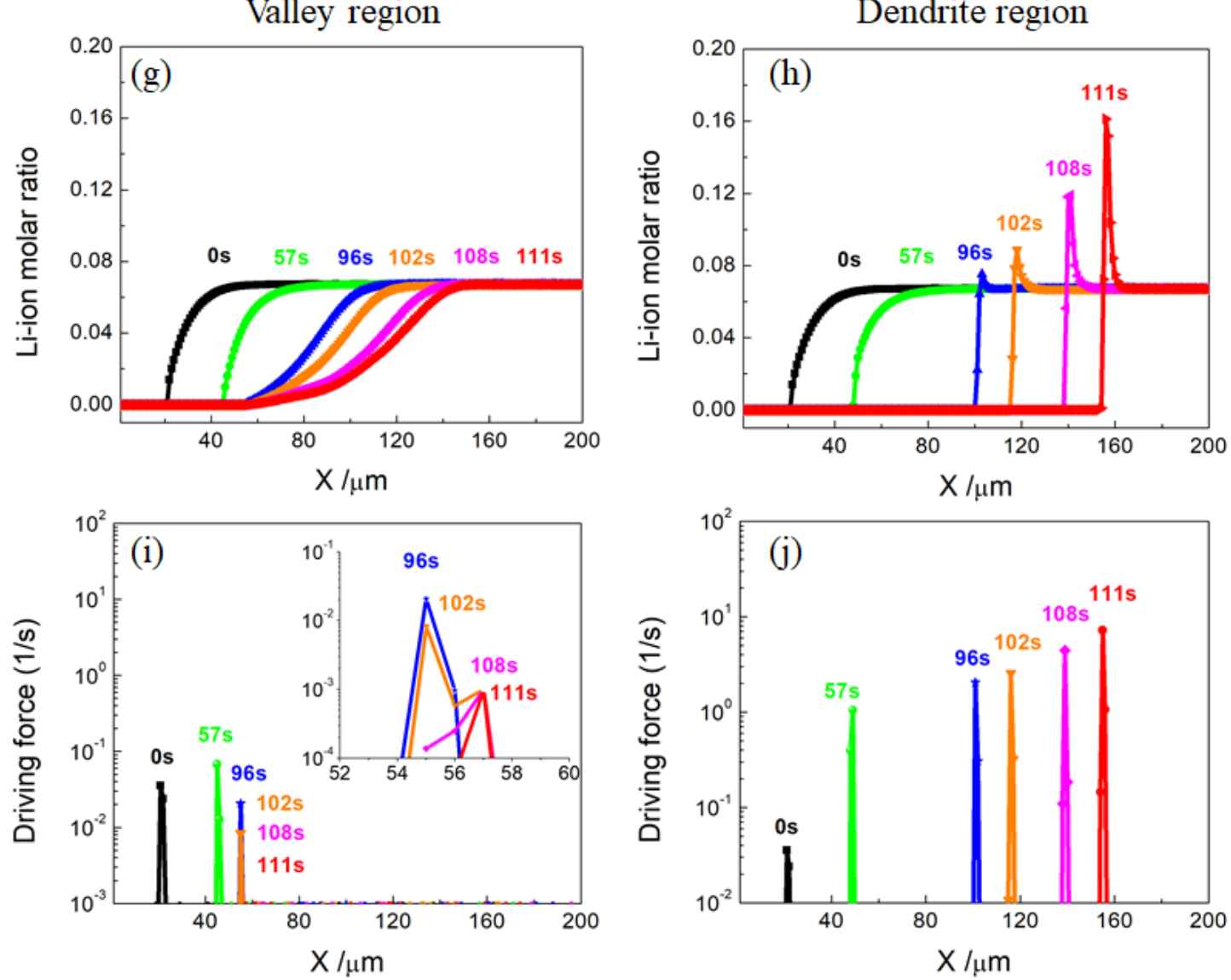

Figure 2: Spatial/temporal distribution of electric potential and driving force. (a)-(c) Evolution of electric potential at $0 \mathrm{~s}, 57 \mathrm{~s}$ and $108 \mathrm{~s}$. (d)-(f) The corresponding spatial distribution of the driving force. (g)-(h) The line plots of Li-ion molar ratio as a function of time in the Valley and Dendrite regions. (i) and (j) The corresponding Line plots of the driving forces in the two regions. 

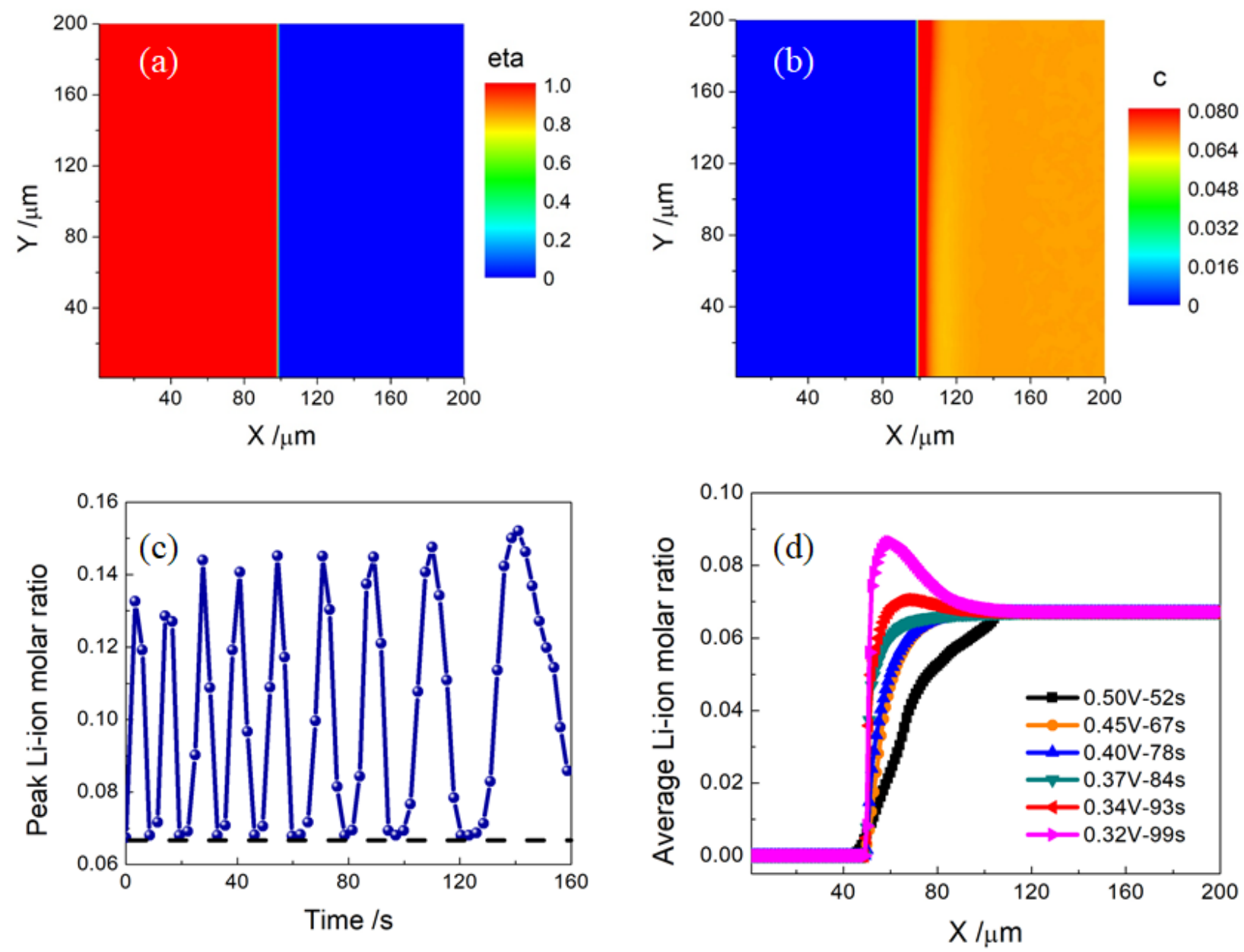

Figure 3: Influence of the applied overpotential. (a) Stable Lithium deposition after $200 \mathrm{~s}$ with an applied overpotential of $-0.32 \mathrm{~V}$. (b) The corresponding spatial distribution of ion concentration. (c) The temporal evolution of the maximum Li-ion concentration. The dashed line shows the bulk concentration. (d) Average Li-ion concentration profile at different applied overpotential after passing the same total current. 
centration gradient). With the increase of reaction rate and decrease of transport rate, the transport rate will be surpassed by the reaction rate, giving rise to a decrease in surface ion concentration. And as it happens, the decrease of surface ion concentration would lead to an increase in ionic transport and decrease of electrochemical reaction. Thus, an iterative feedback system is achieved to guarantee the stable electrodeposition. Importantly, in this case the maximum surface ion concentration is always higher than the concentration of the bulk electrolyte.

Scenario 1:

Small overpotential Stable deposition

$c_{\text {interface }}>c_{\text {bulk }}$

Scenario 2:

Large overpotential Dendrite growth
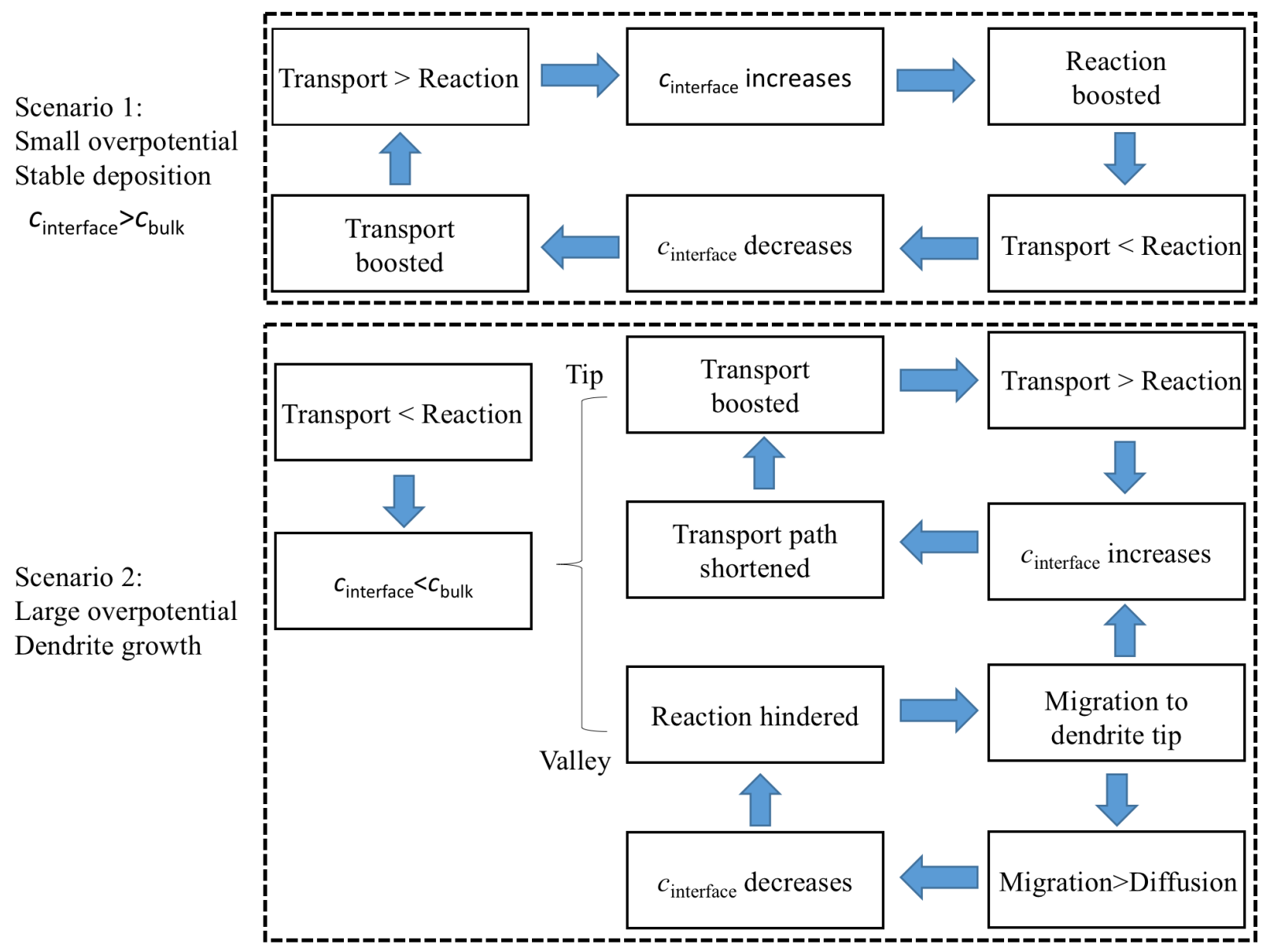

Figure 4: Schematics of the dynamic evolution scenarios at small and large overpotentials (low and high currents).

While under higher overpotential, initially, the electrochemical reaction is faster than the ion transport, resulting in a depletion of ions at the interface. The formation of peaks (dendrites) due to the inhomogeneity of the system will boost the transport of $\mathrm{Li}^{+}$, due to 
not only a larger concentration gradient, but also a higher migration rate from both the source and the surrounding valley regions. This results in a higher transport rate than the reaction rate, leading to a further increase in the surface ion concentration. The increase in surface ion concentration in return boosts the electrochemical reaction and interfacial growth velocity which further shortens the diffusion length. In other surrounding valley regions, the ions are "sucked" by the peak region, the ion concentration keeps dropping which hinders the electrochemical reaction. This effect is purely related to the dimensionality and cannot be captured within 1-dimensional models. The key feature that distinguishes the two scenarios is that the average ion concentration at the interface is always larger than the bulk electrolyte for the lower overpotential case, while vice versa for the higher overpotential. This indicates that even when a surface instability is formed in the first scenario, it will quickly be recovered due to a lower reaction rate with lower concentration at the tip of the surface modulation.

Based on the above understanding, we proposed a new concept "Compositionally graded solid electrolyte" which could potentially suppress the dendrite initiation (given in Supplementary Figure S6). This electrolyte is designed such that a higher Li-ion concentration at the Li-metal anode side and a lower Li-ion concentration at the cathode side. This can be achieved by tuning the polymer/ceramics ratio, or by varying the vacancy/doping concentrations in the solid solutions. Under this design, the initiation of dendrite would be hindered at a reasonable current density since the tips (dendrite) would have a lower concentration than the valley regions, which would decrease the electrochemical reaction rate of the dendrite.

In conclusion, we have developed a hybrid grand potential based nonlinear phase-field model for electrodeposition using fully open source MOOSE package. The dynamic morphological evolution during the lithium electrodeposition process under large and small overpotential is studied, revealing dendrite growth/stable deposition under the two conditions. The concentration, overpotential, driving force distribution is further studied for both cases, showing the critical role of the intimate competitions between ion transport and electrochemical reactions for the formation of dendrites. We further proposed a "compositionally graded 
solid electrolyte" that could potentially lead to the suppression of dendrites. We hope that open-sourcing phase-field models will benefit the community as a whole, and stimulate more theoretical/experimental follow-up studies.

\section{Acknowledgement}

Z.H. and V.V. gratefully acknowledge support from the U.S. Department of Energy, Energy Efficiency and Renewable Energy Vehicle Technologies Office under Award No. deee0007810.

\section{Supporting Information Available}

The following files are available free of charge. All input files for MOOSE simulations are made freely available. Contents in the supporting information: Derivation of the modified diffusion equation; Parameters used in the simulations; Nonlinear dendrite growth kinetics; Branching growth kinetics under high overpotential; Design of "compositionally graded solid electrolyte"; Supplementary videos for the morphology and concentration evolution under applied overpotential of $-0.45 \mathrm{~V}$ and $-0.32 \mathrm{~V}$.

\section{References}

(1) Sripad, S.; Viswanathan, V. Evaluation of Current, Future, and Beyond Li-Ion Batteries for the Electrification of Light Commercial Vehicles: Challenges and Opportunities. J. Electrochem. Soc. 2017, 164, E3635-E3646.

(2) Sripad, S.; Viswanathan, V. Performance Metrics Required of Next-Generation Batteries to Make a Practical Electric Semi Truck. ACS Energy Lett. 2017, 2, 1669-1673.

(3) Guttenberg, M.; Sripad, S.; Viswanathan, V. Evaluating the Potential of Platooning 
in Lowering the Required Performance Metrics of Li-Ion Batteries to Enable Practical Electric Semi-Trucks. ACS Energy Lett. 2017, 2, 2642-2646.

(4) Kerman, K.; Luntz, A.; Viswanathan, V.; Chiang, Y.-M.; Chen, Z. Practical challenges hindering the development of solid state Li ion batteries. J. Electrochem. Soc. 2017, 164, A1731-A1744.

(5) Aurbach, D.; Zinigrad, E.; Cohen, Y.; Teller, H. A short review of failure mechanisms of lithium metal and lithiated graphite anodes in liquid electrolyte solutions. Solid State Ionics 2002, 148, $405-416$.

(6) Cheng, X.-B.; Zhang, R.; Zhao, C.-Z.; Zhang, Q. Toward Safe Lithium Metal Anode in Rechargeable Batteries: A Review. Chem. Rev. 2017, 117, 10403-10473.

(7) Xu, W.; Wang, J.; Ding, F.; Chen, X.; Nasybulin, E.; Zhang, Y.; Zhang, J.-G. Lithium metal anodes for rechargeable batteries. Energy Environ. Sci. 2014, 7, 513-537.

(8) Lin, D.; Liu, Y.; Cui, Y. Reviving the lithium metal anode for high-energy batteries. Nat. Nanotech. 2017, 12, 194-206.

(9) Mullins, W. W.; Sekerka, R. F. Morphological Stability of a Particle Growing by Diffusion or Heat Flow. J. Appl. Phys. 1963, 34, 323-329.

(10) Mullins, W. W.; Sekerka, R. F. Stability of a Planar Interface During Solidification of a Dilute Binary Alloy. J. Appl. Phys. 1964, 35, 444-451.

(11) Monroe, C.; Newman, J. Dendrite Growth in Lithium/Polymer Systems: A Propagation Model for Liquid Electrolytes under Galvanostatic Conditions. J. Electrochem. Soc. 2003, 150, A1377-A1384.

(12) Monroe, C.; Newman, J. The Effect of Interfacial Deformation on Electrodeposition Kinetics. J. Electrochem. Soc. 2004, 151, A880-A886. 
(13) Monroe, C.; Newman, J. The Impact of Elastic Deformation on Deposition Kinetics at Lithium/Polymer Interfaces. J. Electrochem. Soc. 2005, 152, A396-A404.

(14) Tikekar, M. D.; Archer, L. A.; Koch, D. L. Stabilizing electrodeposition in elastic solid electrolytes containing immobilized anions. Sci. Adv. 2016, 2, 1600320.

(15) Ahmad, Z.; Viswanathan, V. Stability of Electrodeposition at Solid-Solid Interfaces and Implications for Metal Anodes. Phys. Rev. Lett. 2017, 119, 056003.

(16) Ahmad, Z.; Viswanathan, V. Role of anisotropy in determining stability of electrodeposition at solid-solid interfaces. Phys. Rev. Materials 2017, 1, 055403.

(17) Chen, L.-Q. Phase-Field Models for Microstructure Evolution. Annu. Rev. Mater. Res. 2002, 32, 113-140.

(18) Boettinger, W. J.; Warren, J. A.; Beckermann, C.; Karma, A. Phase-Field Simulation of Solidification. Annu. Rev. Mater. Res. 2002, 32, 163-194.

(19) Chen, L.; Zhang, H. W.; Liang, L. Y.; Liu, Z.; Qi, Y.; Lu, P.; Chen, J.; Chen, L.Q. Modulation of dendritic patterns during electrodeposition: A nonlinear phase-field model. J. Power Sources 2015, 300, 376 - 385.

(20) Cogswell, D. A. Quantitative phase-field modeling of dendritic electrodeposition. Phys. Rev. E 2015, 92, 011301.

(21) Liang, L.; Chen, L.-Q. Nonlinear phase field model for electrodeposition in electrochemical systems. Appl. Phys. Lett. 2014, 105, 263903.

(22) Liang, L.; Qi, Y.; Xue, F.; Bhattacharya, S.; Harris, S. J.; Chen, L. Q. Nonlinear phase-field model for electrode-electrolyte interface evolution. Phys. Rev. E 2012, 86, $1-5$.

(23) Ely, D. R.; Jana, A.; Garca, R. E. Phase field kinetics of lithium electrodeposits. J. Power Sources 2014, 272, $581-594$. 
(24) Bazant, M. Z. Theory of Chemical Kinetics and Charge Transfer based on Nonequilibrium Thermodynamics. Acc. Chem. Res. 2013, 46, 1144-1160.

(25) Kitchin, J. R. Examples of Effective Data Sharing in Scientific Publishing. ACS Catal. 2015, 5, 3894-3899.

(26) Plapp, M. Unified derivation of phase-field models for alloy solidification from a grandpotential functional. Phys. Rev. E 2011, 84, 031601.

(27) Gaston, D.; Newman, C.; Hansen, G.; Lebrun-Grandi, D. MOOSE: A parallel computational framework for coupled systems of nonlinear equations. Nucl. Eng. Des. 2009, 239, $1768-1778$.

(28) Tonks, M. R.; Gaston, D.; Millett, P. C.; Andrs, D.; Talbot, P. An object-oriented finite element framework for multiphysics phase field simulations. Comput. Mater. Sci. 2012, $51,20-29$.

(29) Yurkiv, V.; Foroozan, T.; Ramasubramanian, A.; Shahbazian-Yassar, R.; Mashayek, F. Phase-field modeling of solid electrolyte interface (SEI) influence on Li dendritic behavior. Electrochim. Acta 2018, 265, 609 - 619. 


\title{
Supporting Information:
}

\section{Phase-field simulations of lithium dendrite growth with open-source software}

\author{
Zijian Hong and Venkatasubramanian Viswanathan* \\ Department of Mechanical Engineering, Carnegie Mellon University, Pittsburgh, \\ Pennsylvania 15213, USA \\ E-mail: venkvis@cmu.edu
}

Derivation of the modified diffusion equation. The diffusion equation is derived starting from the mass conservation law:

$$
\frac{\partial C}{\partial t}=-\nabla \cdot J
$$

where $C, t$ and $J$ are the volume concentration, evolution time and flux of lithium species, respectively. The lithium species include lithium ion $\left(\mathrm{Li}^{+}\right)$and lithium metal. Note that only lithium species are conserved rather than lithium ion, due to the electrochemical reaction at the interface. The volume concentration $C$ has the contributions from the electrode and electrolyte phases:

$$
C=C^{l}(1-h(\xi))+C^{s} h(\xi) .
$$

where $C^{l}$ and $C^{s}$ are the volume concentration of lithium species in the electrolyte and electrode phases, respectively. $h(\xi)$ is the interpolation function which is given in the main 
text. Combining Equations 1 and 2, we can get:

$$
\frac{\partial C}{\partial t}=h(\xi)\left[\frac{\partial C^{s}}{\partial t}-\frac{\partial C^{l}}{\partial t}\right]+\frac{\partial h(\xi)}{\partial t}\left[C^{s}-C^{l}\right]=-\nabla . J
$$

Assuming that the diffusivity of the Li-metal is much smaller than the diffusivity of the lithium ion, the flux of lithium species can be written as:

$$
J=-\frac{D C_{L i^{+}}}{R T}[\nabla \mu+n F \nabla \phi]
$$

where $D$ and $C_{L i^{+}}$are the diffusivity and volume concentration of $\mathrm{Li}^{+}$, respectively. $R, T, n, F$ are the gas constant, temperature, number of electrons in the ion and Faraday constant, respectively. $\mu$ is the chemical potential of lithium. $\phi$ is the local applied overpotential. Combine Equations 3 and 4 together and divide by the site density of the electrolyte $C_{m}^{l}$, one can deduce:

$$
h(\xi)\left[\frac{\partial c^{s}}{\partial t} \frac{C_{m}^{s}}{C_{m}^{l}}-\frac{\partial c^{l}}{\partial t}\right]+\frac{\partial h(\xi)}{\partial t}\left[c^{s} \frac{C_{m}^{s}}{C_{m}^{l}}-c^{l}\right]=\nabla \cdot \frac{D c_{L i^{+}}}{R T}[\nabla \mu+n F \nabla \phi]
$$

Rewriting equation 5 in terms of chemical potential $\mu$, we get:

$$
\chi \frac{\partial \mu}{\partial t}=\nabla \cdot \frac{D c_{L i^{+}}}{R T}[\nabla \mu+n F \nabla \phi]-\frac{\partial h(\xi)}{\partial t}\left[c^{s} \frac{C_{m}^{s}}{C_{m}^{l}}-c^{l}\right]
$$

where the susceptibility factor $\chi=\frac{\partial c^{l}}{\partial \mu}[1-h(\xi)]+\frac{\partial c^{s}}{\partial \mu} h(\xi) \frac{C_{m}^{s}}{C_{m}^{l}}$.

The molar ratio of electrolyte and electrode phases are related to the chemical potential, ${ }^{1}$ given by,

$$
c^{l, s}=\frac{\exp \left[\frac{\left(\mu-\epsilon^{l, s}\right)}{R T}\right]}{1+\exp \left[\frac{\left(\mu-\epsilon^{l, s}\right)}{R T}\right]}
$$

where $\epsilon^{l, s}=\mu^{0 l, 0 s}-\mu^{0 N}$ is the difference in the chemical potential of lithium and neutral components on the electrolyte/electrode phase at initial equilibrium state. The lithium ion molar ratio $c_{L i^{+}}$is related to the electrolyte molar ratio $c^{l}$ : 


$$
c_{L i^{+}}=c^{l}(1-h(\xi))=\frac{\exp \left[\frac{\left(\mu-\epsilon^{l}\right)}{R T}\right]}{1+\exp \left[\frac{\left(\mu-\epsilon^{l}\right)}{R T}\right]}(1-h(\xi))
$$

Equation 6 can be rearranged to obtain the Equation 3 in the main text:

$$
\frac{\partial \mu}{\partial t}=\frac{1}{\chi}\left[\nabla \cdot \frac{D c_{L i^{+}}}{R T}(\nabla \mu+n F \nabla \phi)-\frac{\partial h(\xi)}{\partial t}\left(c^{s} \frac{C_{m}^{s}}{C_{m}^{l}}-c^{l}\right)\right]
$$

Note that this equation is identical to model developed by Cogswell ${ }^{1}$ (see Equation 9 in ref. 1) only when $C_{m}^{s}=C_{m}^{l}$. This assumption has been explicitly made in the original paper by Plapp $^{2}$ in deriving the diffusion equation. However, this is not valid in the present study.

Parameters used in the simulation The electrolyte is assumed to be $1 \mathrm{M} \mathrm{LiPF}_{6}$ dissolved in $\mathrm{EC} / \mathrm{DMC}$ (1:1 volume ratio) solutions. The kinetic coefficient $L_{\eta}$ is calculated using the following relationship: $L_{\eta}=i_{0} \frac{V_{m} \gamma}{F \kappa} .{ }^{1}$ The barrier height $\mathrm{W}$ and gradient coefficient $\kappa$ are related to the surface tension $\gamma$ and the interfacial thickness $\delta: W=12 \frac{\gamma}{\delta}, \kappa=\frac{3 \gamma \delta}{2} .{ }^{1}$ The site density of the electrolyte can be computed by the density $(1.3 \mathrm{~g} / \mathrm{L})$ and molar mass (90 g/mol) of the electrolyte. All the parameters used in the current phase-field model are shown in the following table:

Table S1: Phase-field simulation parameters

\begin{tabular}{||ccccc||}
\hline Variable name & Symbol & Real value & Normalized value & Source \\
\hline \hline Interfacial mobility & $L_{\sigma}$ & $2.5 \times 10^{-6} \mathrm{~m}^{3} /(J \times s)$ & 6.25 & {$[3]$} \\
Limiting current & $i_{0}$ & $3 \mathrm{~mA} / \mathrm{cm}^{2}$ & 30 & {$[4]$} \\
Kinetic coefficient & $L_{\eta}$ & $0.001 / \mathrm{s}$ & 0.001 & Computed \\
Electrons transfered & $n$ & 1 & 1 & - \\
Surface tension & $\gamma$ & $0.556 \mathrm{~J} / \mathrm{m}^{2}$ & 0.22 & {$[5]$} \\
Interface thickness & $\delta$ & $1 \mu \mathrm{m}$ & 1 & Estimated \\
Transfer coefficient & $\alpha$ & 0.5 & 76.4 & {$[3]$} \\
Site density of electrode & $C_{m}^{s}$ & $7.64 \times 10^{4} \mathrm{~mol} / \mathrm{m}^{3}$ & 14.4 & Computed \\
Site density of electrolyte & $C_{m}^{l}$ & $1.44 \times 10^{4} \mathrm{~mol} / \mathrm{m}^{3}$ & $10^{7}$ & {$[3]$} \\
Conductivity of electrode & $\sigma^{s}$ & $10^{7} \mathrm{~S} / \mathrm{m}$ & 1.19 & {$[6]$} \\
Conductivity of electrolyte & $\sigma^{l}$ & $1.19 \mathrm{~S} / \mathrm{m}^{7}$ & 319.7 & {$[6]$} \\
Diffusivity of Lithium ion & $\mathrm{D}$ & $3.197 \times 10^{-10} \mathrm{~m}^{2} / \mathrm{s}$ & & \\
\hline
\end{tabular}

Nonlinear dendrite growth kinetics The interface movement under an applied overpo- 
tential of $-0.45 \mathrm{~V}$ is tracked by plotting the temporal evolution of order parameter $\xi$ along X-direction, as shown in Figure S1a. A sharp interface is observed where the order parameter changes abruptly from 1 to 0 across the interface, which is due to the fact that the interface thickness is much smaller than the system size. The interface moves by $\sim 30 \mu \mathrm{m}$ after $57 \mathrm{~s}$, while a further movement of $\sim 90 \mu \mathrm{m}$ is shown after another $51 \mathrm{~s}$. The position of the phase boundary is plotted with respect to time, showing a nearly linear growth before $60 \mathrm{~s}$, followed by a rapid nonlinear growth thereafter (Figure S1b). The interface velocity is further computed, which is almost constant before $60 \mathrm{~s}$ and then increases exponentially over time. This clearly demonstrated that the dendrite growth is highly nonlinear, which could deteriorate the performance of the battery almost immediately.
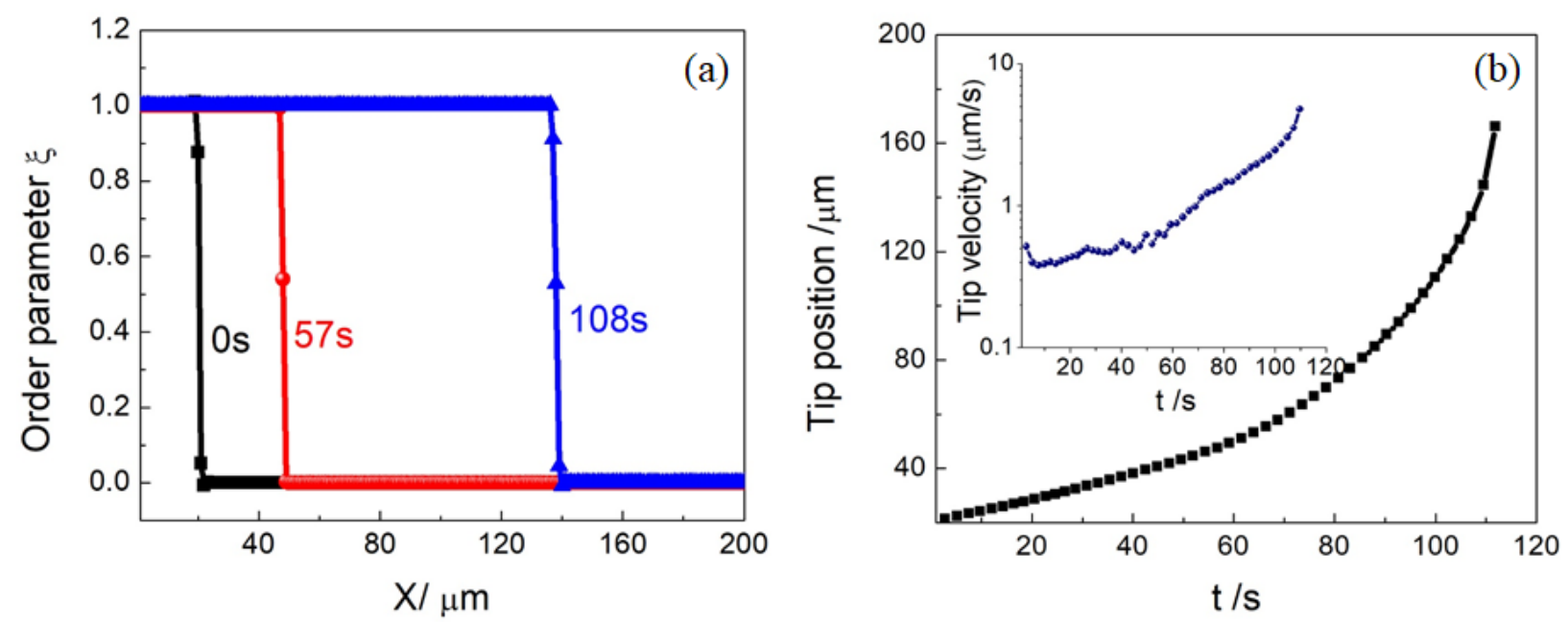

Figure S1: (a) Interface movement (b) and time dependent dendrite growth velocity

The spatial distribution of the electric potential after the formation of dendrites is analyzed in detail (Figure S2). Figure S2a shows the 2-dimensional distribution of the electric potential, which indicates a nearly constant potential at the electrode side (shown in blue), while gradually increases to zero at right boundary deep in the electrolyte. The comparison of the potential distribution between the tip and valley regions is plotted in Figure S2b, which shows a nonlinear potential change near the interfaces of both the valley and tip regions. Meanwhile, the overpotential profile is more flat for the valley region than the tip 
region. We further plot the initial electric overpotential distribution along X-direction as a comparison, which gives a linear increase from the electrode/electrolyte interface to the electrolyte (Figure S2d ). Meanwhile, the overpotential distribution along Y-direction (Figure S2c) demonstrates a deep peak for the dendrite tip, resulting in an electric field from the valley to the tip.
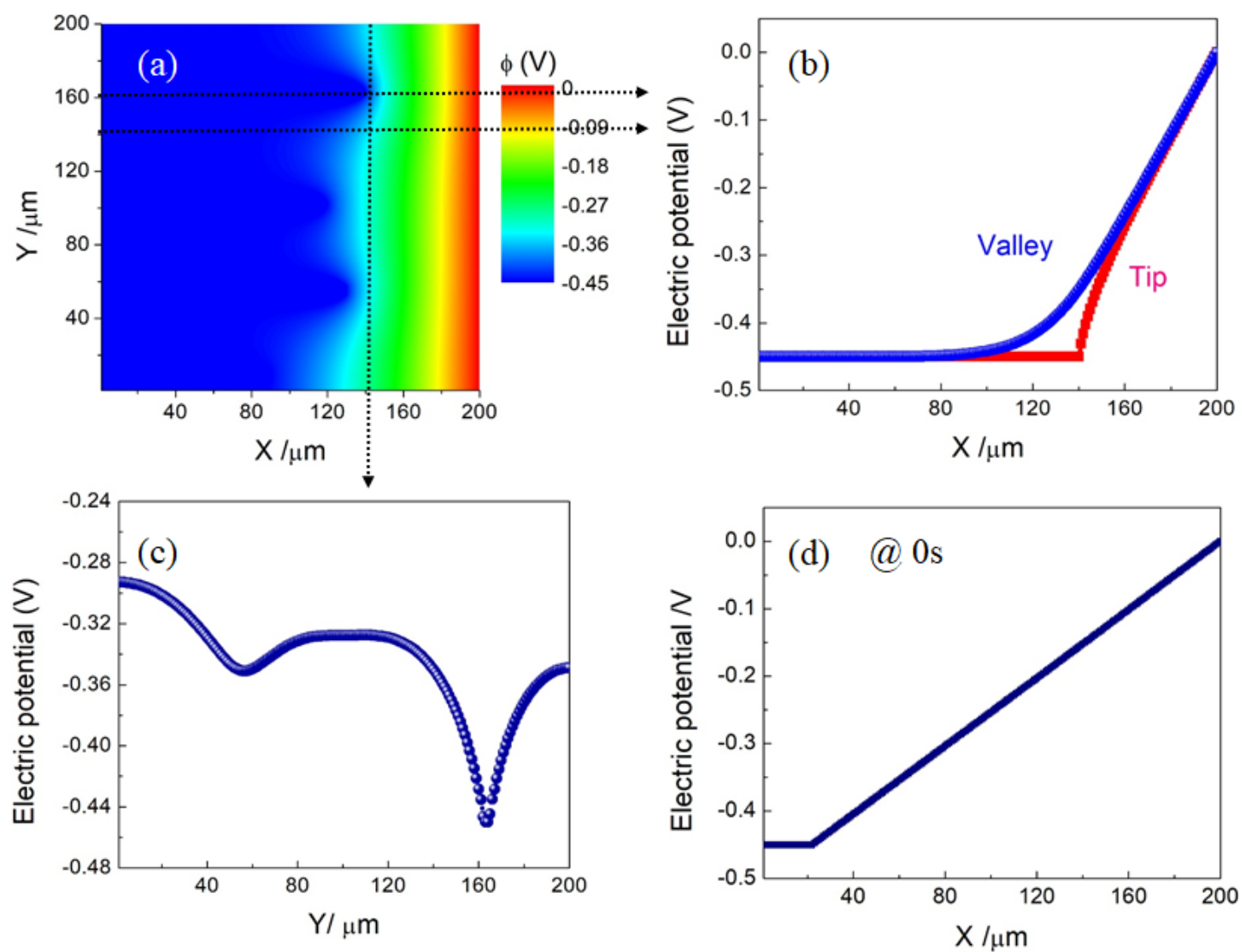

Figure S2: Spatial distribution of electric overpotential (a) potential distribution after the formation of dendrite (b) line plot of the potential distribution for both tip and valley regions (c) line plot of the potential distribution along Y-direction cutting through dendrite tips (d) initial potential distribution along X-direction.

The electric field distribution is calculated by numerical differentiation of the electric potential, as depicted in Figure S3. It can be seen that the electric field in the electrode region is minimal, as the electrode metal has a much larger conductivity than the electrolyte. Interestingly, the electric field near the valley region between the dendrites is also very small, this is consistent with the flat electrical overpotential distribution for the valley region shown 
in Figure S2b. The magnified view (Figure S3b) clearly demonstrates that the electric field is highly localized surrounding the dendrite tip, causing strong ion migration from the neighboring regions.
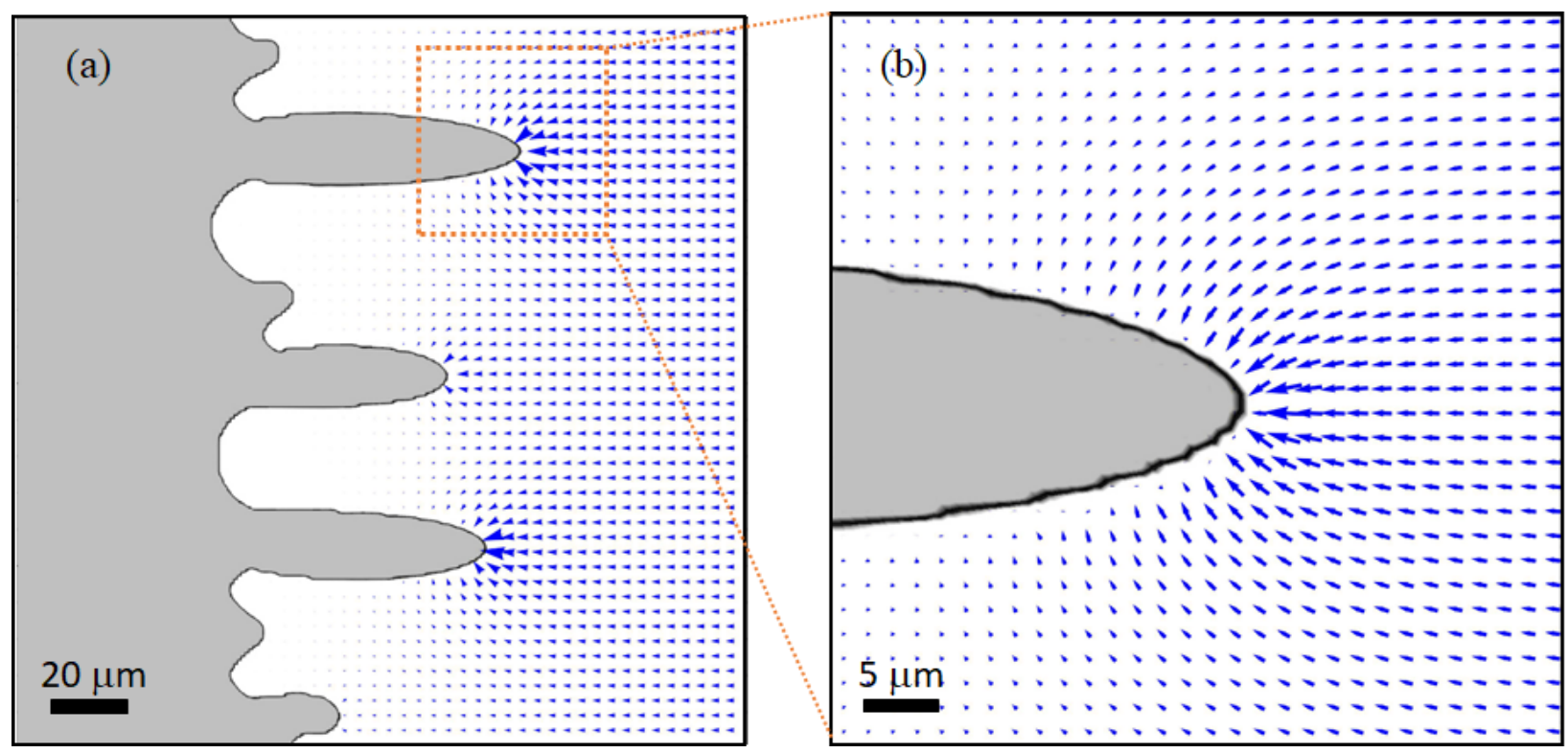

Figure S3: Overlay of electric field vector with the morphology. (a) In the whole simulation volume (b) magnified view in the vicinity of the dendrite tip

Branching growth kinetics under high overpotential. The growth of side branch (treelike) structures is observed in several previous phase-field studies. ${ }^{1,3,7}$ Here, we also show that the side branching can occur at high overpotentials, e.g., -0.5 V (Figure S4). Initially, after $25 \mathrm{~s}$, the interfacial instability shows up, with very fine nuclei. It can be seen that the critical size of the nuclei is much smaller at larger overpotential, which is consistent with experimental observations. ${ }^{8}$ This can be easily understood as at higher overpotential, even small perturbation can cause the surface instability and overcome the nucleation barrier. After $45 \mathrm{~s}$, some of the nuclei are growing to form dendrites (Figure S4b), while most of the nuclei growth are hindered. Eventually, only one of these dendrites grow at an overwhelming speed, penetrating through the electrolyte (Figure S4c). Interestingly, with the growth of this long dendrite, some side branches are observed, forming a hyper-branched dendritic structure. The corresponding concentration profile is given in Figures S4(d)-(e). It is shown 
that a broad interface is formed similar to the previous case under $-0.45 \mathrm{~V}$. After forming the long dendrite, as shown in Figure S4f, a much higher concentration can be found near the dendrite tip.
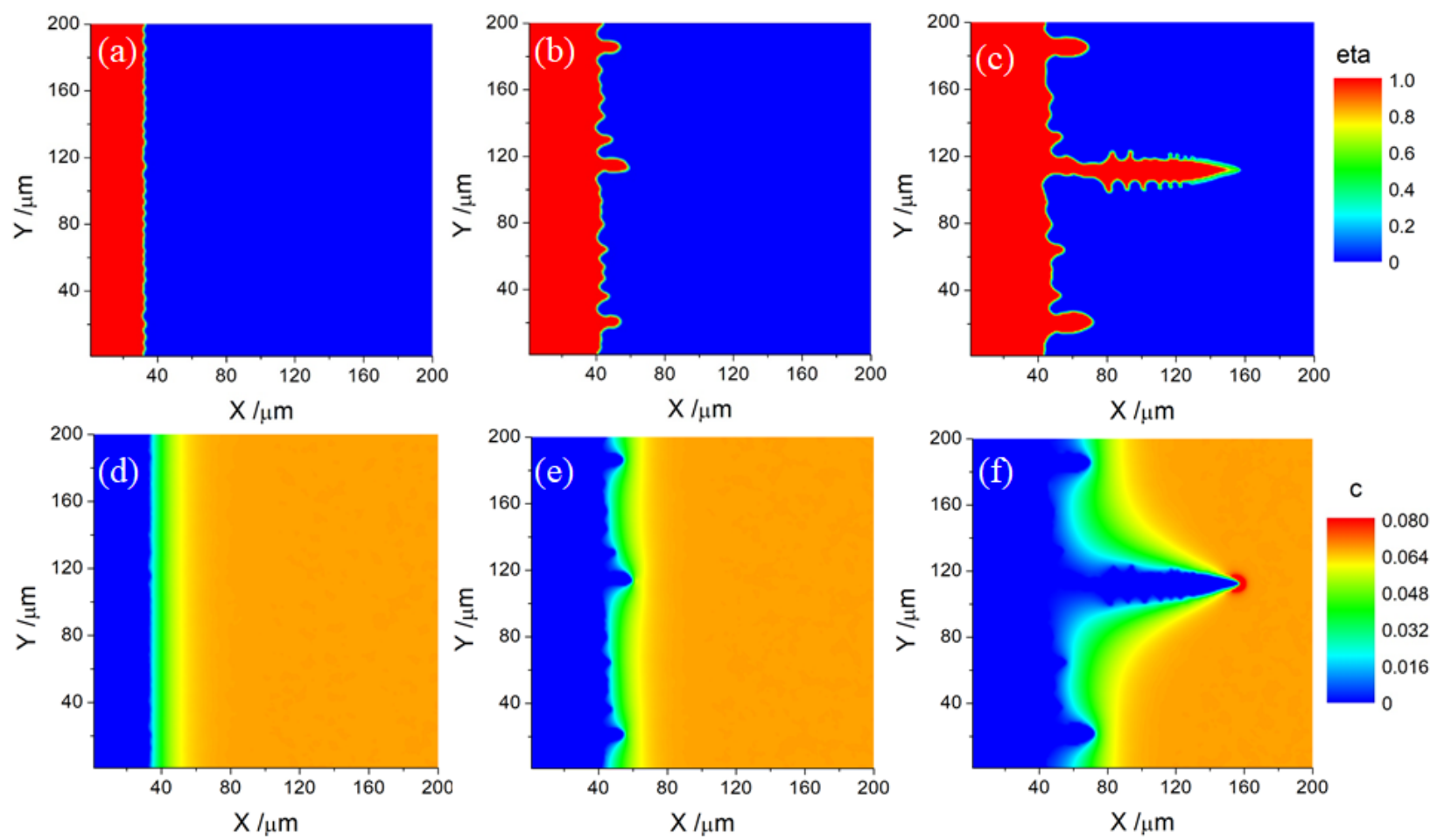

Figure S4: Dynamic morphological evolution under large overpotential of $-0.5 \mathrm{~V}$ (a) after $25 \mathrm{~s}$ (b) after $45 \mathrm{~s}$ (c) after $56 \mathrm{~s}$. (d)-(f) the corresponding spatial distribution of Li-ion concentration.

The spatial distributions of applied overpotential and driving force are displayed in Figure S5. The overpotential distribution is almost linear from the electrode/electrolyte interface to electrolyte before the dendrites grow, while a wedge-like distribution is shown after the formation of the long dendrite (Figure S5c). This kind of potential distribution will also give large electric field towards the dendrite tip. The corresponding driving force is plotted in Figures S5 (d)-(f). A larger driving force can be clearly found at the interface regions. While interestingly, after the formation of the long dendrite, the region with side branches also has much larger driving force, indicating that the side branches can also grow larger in this case.

Based on the insights from the simulations, we propose a "compositionally graded solid 

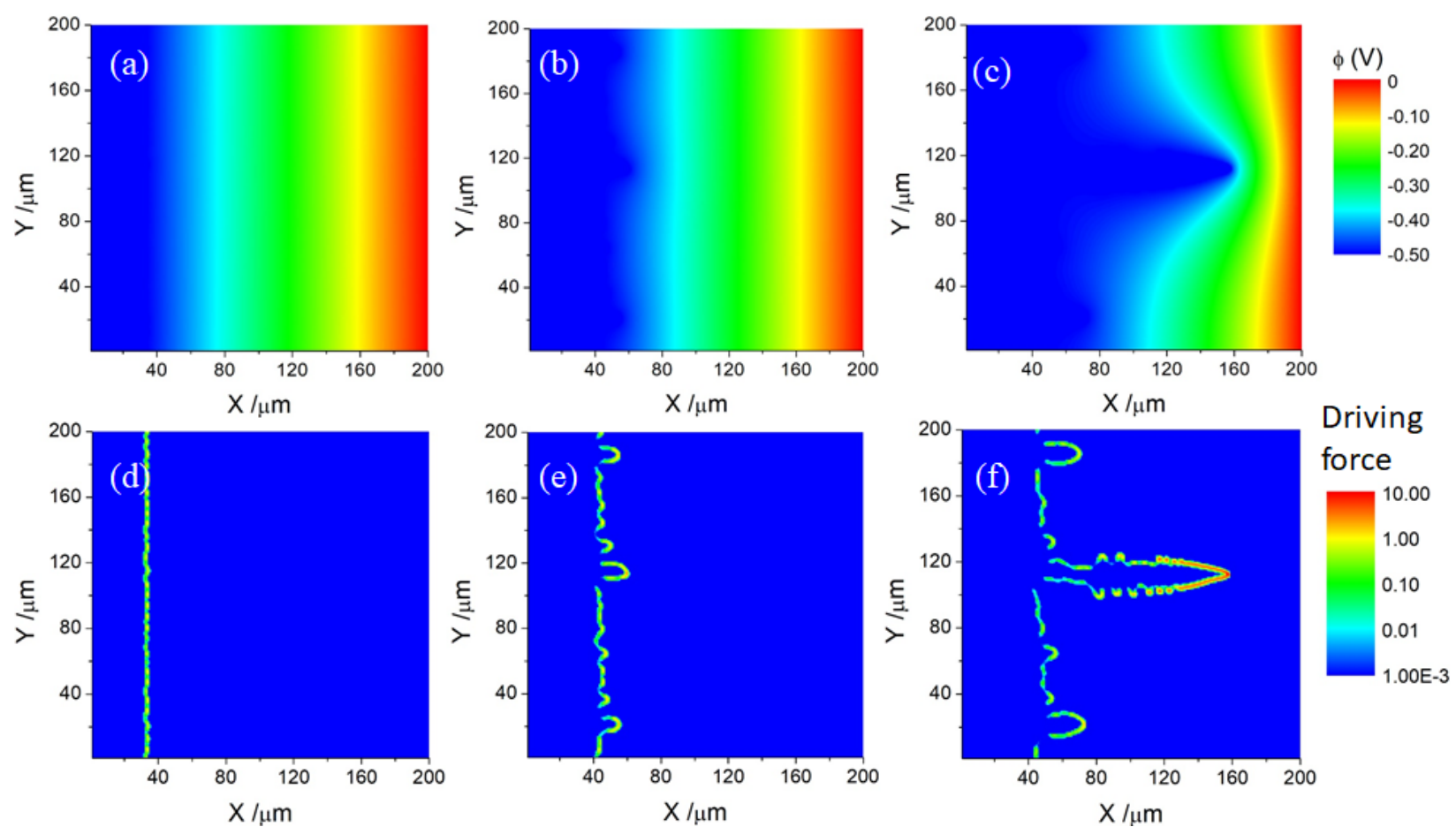

Figure S5: Spatial/temporal distribution of electric potential and driving force at large overpotential. (a)-(c) Evolution of electric potential at $25 \mathrm{~s}, 45 \mathrm{~s}$ and $56 \mathrm{~s}$. (d)-(f) The corresponding spatial distribution of the driving force.

electrolyte" as a potential approach to suppress the dendrite initiation, as shown in Figure S6. The electrolyte can be designed such that it has a higher Li-ion concentration close to the Li-metal anode side while keeping a reasonable relatively lower Li-ion concentration in the vicinity of the cathode side. As the deposition goes on, even if small nuclei are forming, the tip region will have a lower ion concentration than the valley region. Since the reaction rate is directly proportional to the ion concentration, as a result, it can be expected that the electrochemical reaction rate at the tip will be lower than at the valley region, which will prevent the growth of the dendrite at the initial stage. This design may be realized by tuning the doping levels of solid electrolytes (e.g., increasing $\mathrm{x}$ in $\mathrm{Li}_{7-3 x} \mathrm{Al}_{x} \mathrm{La}_{3} \mathrm{Zr}_{2} \mathrm{O}_{12}$ from the anode to cathode), or by varying the lithium containing ceramic to polymer ratio.

\section{Supplementary videos available.}

Supplementary video 1: Dendrite growth under an applied overpotential of $-0.45 \mathrm{~V}$.

Supplementary video 2: Corresponding Lithium-ion concentration evolution @-0.45 V. 

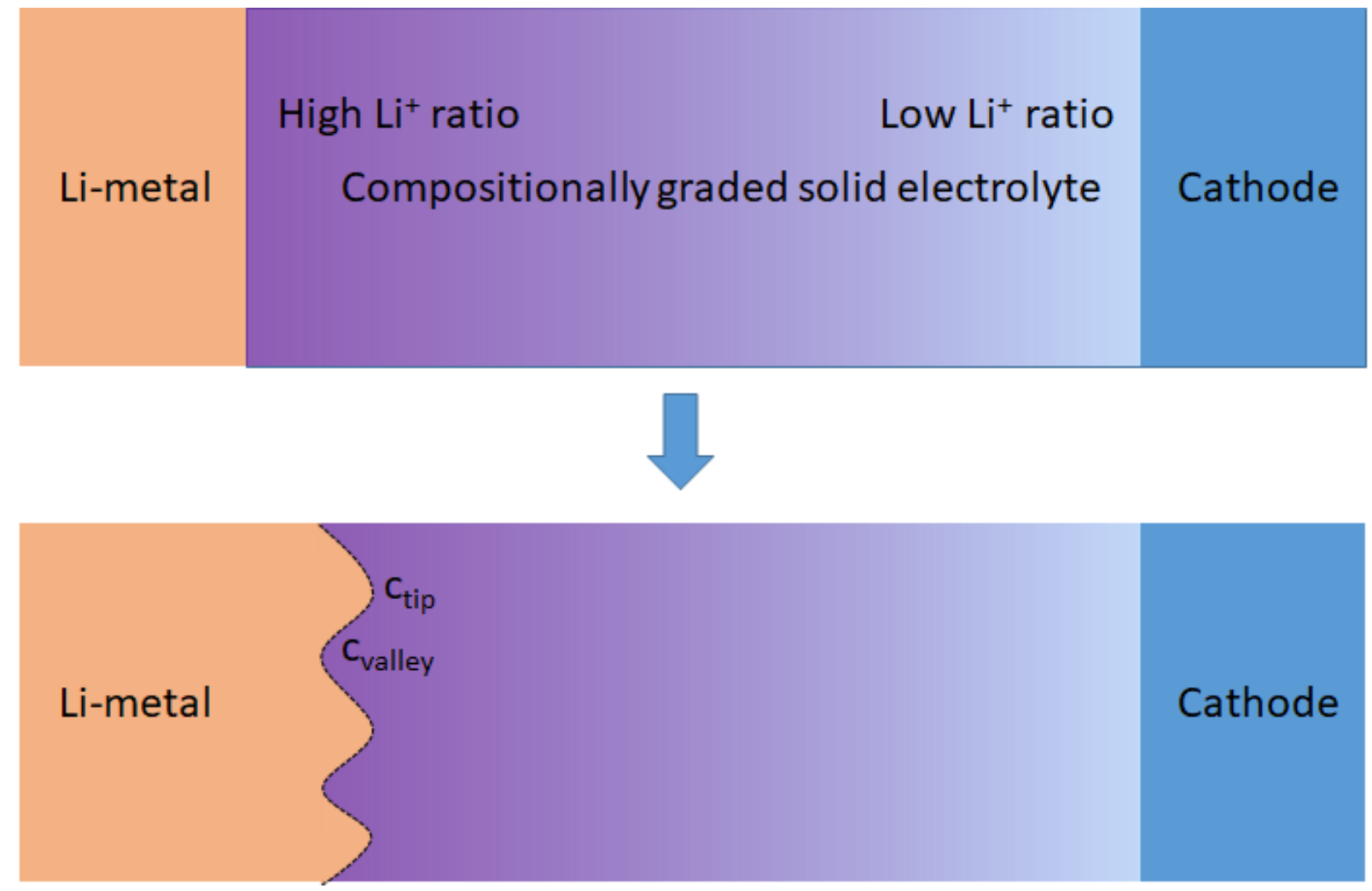

Concentration: $c_{t i p}<c_{\text {valley }}$ Deposition rate: $R_{\text {tip }}<R_{\text {valley }}$ Dendrite suppress!

Figure S6: Schematics of the proposed design "compositionally graded solid electrolyte" 
Supplementary video 3: Stable deposition under an applied overpotential of -0.32 V.

Supplementary video 4: Corresponding Lithium-ion concentration evolution @-0.32 V. 


\section{References}

(1) Cogswell, D. A. Quantitative phase-field modeling of dendritic electrodeposition. Phys. Rev. E 2015, 92, 011301.

(2) Plapp, M. Unified derivation of phase-field models for alloy solidification from a grandpotential functional. Phys. Rev. E 2011, 84, 031601.

(3) Chen, L.; Zhang, H. W.; Liang, L. Y.; Liu, Z.; Qi, Y.; Lu, P.; Chen, J.; Chen, L.Q. Modulation of dendritic patterns during electrodeposition: A nonlinear phase-field model. J. Power Sources 2015, 300, 376 - 385.

(4) Monroe, C.; Newman, J. Dendrite Growth in Lithium/Polymer Systems: A Propagation Model for Liquid Electrolytes under Galvanostatic Conditions. J. Electrochem. Soc. 2003, 150, A1377-A1384.

(5) Vitos, L.; Ruban, A.; Skriver, H.; KollÂąr, J. The surface energy of metals. Surf. Sci. 1998, 411, $186-202$.

(6) Valøen, L. O.; Reimers, J. N. Transport Properties of LiPF6-Based Li-Ion Battery Electrolytes. J. Electrochem. Soc. 2005, 152, A882-A891.

(7) Yurkiv, V.; Foroozan, T.; Ramasubramanian, A.; Shahbazian-Yassar, R.; Mashayek, F. Phase-field modeling of solid electrolyte interface (SEI) influence on Li dendritic behavior. Electrochim. Acta 2018, 265, 609 - 619.

(8) Pei, A.; Zheng, G.; Shi, F.; Li, Y.; Cui, Y. Nanoscale Nucleation and Growth of Electrodeposited Lithium Metal. Nano Lett. 2017, 17, 1132-1139. 


\section{Graphical TOC Entry}

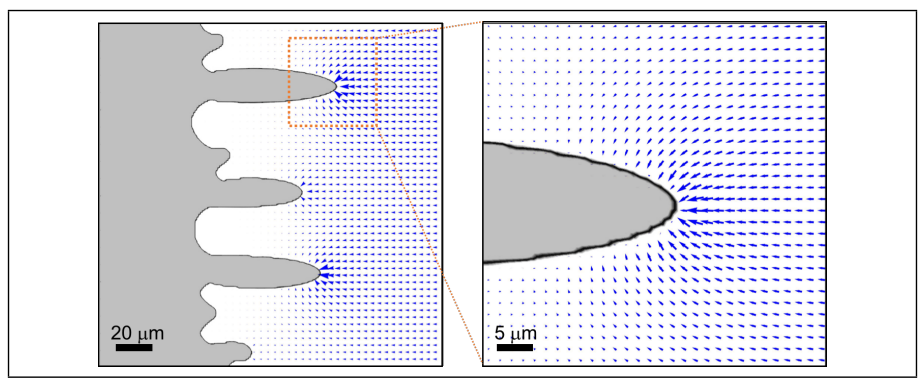

